\title{
ESTRUCTURAS ORGANIZACIONALES Y ADAPTACIÓN A LAS CONDICIONES CAMBIANTES DEL ENTORNO: RETOS E IMPLICACIONES
}

Laura Yaritza Gualdrón Prieto ${ }^{1}$, Johana Marcela Acosta Romero², Luz Esperanza Bohórquez Arévalo ${ }^{3}$

${ }^{1}$ Estudiante de Ingeniería Industrial. Correo electrónico: lauragual12@gmail.com ${ }^{2}$ Estudiante de Ingeniería Industrial

${ }^{3}$ Doctora en Ciencias de la Dirección. Profesora titular

Universidad Distrital Francisco José de Caldas Bogotá, Colombia

Fecha de recibido:15 de marzo del $2017 \quad$ Fecha de aprobado: 10 de agosto del 2017

Cómo citar este artículo: L. Y. Gualdrón-Prieto, J. M. Acosta-Romero y L. E. Bohórquez-Arévalo, "Estructuras organizaciones y adaptación a las condiciones cambiantes del entorno: retos e implicaciones", Ingeniería Solidaria, vol. 13, n. ${ }^{\circ}$ 23, pp. 106-122, Sept. 2017. doi: https://doi.org/10.16925/in.v23i13.1983

Resumen: Introducción: en este artículo, realizado en el 2017 en la Universidad Distrital Francisco José de Caldas, se identifican algunas de las características, ventajas y limitaciones de las estructuras organizacionales que buscan incrementar la capacidad de adaptación a las condiciones cambiantes del entorno. Metodología: a) se argumenta que las organizaciones empresariales son sistemas de procesamiento de información; b) se muestra que las estructuras organizacionales pueden ser comprendidas como redes por las que circula la información; c) se realiza la revisión de estado de literatura de investigaciones publicadas en el periodo 2016-2010; de estas se realiza un análisis metódico y exhaustivo de las tendencias, avances, metodología, investigaciones futuras, resultados y conclusiones en los veinte principales journals de la categoría Business, Management and Accounting de Scimago, y d) se plantean retos de la investigación y problemas aún no superados. Resultados: se identifican las características, ventajas y limitaciones de las estructuras organizacionales alternativas al control jerárquico. Se proyectan futuros trabajos de investigación en el diseño de estructuras organizacionales que incrementen la capacidad de adaptación del sistema a las condiciones cambiantes del entorno. Conclusiones: la capacidad de adaptación ligada al procesamiento de altos volúmenes de información es un reto al que se enfrentan las organizaciones con mayor dificultad diariamente, y que podría afrontar de una forma satisfactoria implementando estructuras flexibles.

Palabras clave: estructura organizacional, flexibilidad, gobernanza, heterarquía, redes. 


\title{
Organizational STRUCTURES AND ADAPTATION TO CHANGING ENVIRONMENTAL CONDITIONS: Challenges AND IMPLiCATIONS
}

\begin{abstract}
Introduction: This article, prepared in 2017 at the Universidad Distrital Francisco José de Caldas, identifies some of the characteristics, advantages, and limitations of organizational structures that seek to increase the ability to adapt to changing environmental conditions. Methodology: a) it is argued that business organizations are information processing systems; b) it is shown that organizational structures can be understood as information circulating networks; c) the research literature published in the 2010-2016 period is reviewed; it was methodically and exhaustively analyzed for trends, advances, methodology, future research, results, and conclusions in the twenty main journals of the Business, Management and Accounting category of Scimago; and d) research challenges and problems not yet overcome are set out. Results: The characteristics, advantages, and limitations of organizational structures other than hierarchical control are identified. Future research projects in the design of organizational structures that increase the ability of the system to adapt to changing environmental conditions are planned. Conclusions: The ability to adapt linked to the processing of high volumes of information is a challenge faced daily by organizations which could be satisfactorily addressed by implementing flexible structures.
\end{abstract}

Keywords: organizational structure, flexibility, governance, heterarchy, networks.

\section{ESTRUTURAS ORGANIZACIONAIS E}

\section{ADAPTAÇÃo Às CONDIÇÕES VARIÁVEIS DO ENTORNO: DESAFIOS E IMPLICAÇÕES}

Resumo: Introdução: neste artigo, realizado em 2017, na Universidade Distrital Francisco José de Caldas, são identificadas algumas características, vantagens e limitações das estruturas organizacionais que buscam aumentar a capacidade de adaptação às condições variáveis do entorno. Metodologia: a) argumenta-se que as organizações empresariais são sistemas de processamento de informação; b) mostra-se que as estruturas organizacionais podem ser compreendidas como redes pelas quais a informação circula; c) é realizada a revisão de estado da literatura de pesquisas publicadas no período de 2010 a 2016; a partir deles, é realizada uma análise metódica e exaustiva das tendências, avanços, metodologia, futuras pesquisas, resultados e conclusões nas vinte principais publicações da categoria Negócios, Administração e Contabilidade de Scimago; d) novos desafios da pesquisa e problemas ainda não superados são apresentados. Resultados: as características, vantagens e limitações das estruturas organizacionais alternativas ao controle hierárquico são identificadas. Futuros trabalhos de pesquisa na criação de estruturas organizacionais que aumentem a capacidade de adaptação do sistema às condições variáveis do entorno. Conclusões: a capacidade de adaptação ligada ao processamento de altos volumes de informação é um desafio que as organizações enfrentam com mais dificuldade diariamente e que poderiam afrontar de forma satisfatória implementando estruturas flexíveis.

Palavras-chave: estrutura organizacional, flexibilidade, governança, heterarquia, redes. 


\section{Introducción}

La importancia de la adaptabilidad organizacional a las condiciones cambiantes del entorno ha sido destacada en la literatura como factor clave para el desempeño empresarial [1], [2], [3]. Un amplio porcentaje de investigaciones ha enfatizando la relevancia de las estructuras organizacionales en la capacidad de adaptación del sistema [4], debido a que las estructuras hacen referencia a las formas como interactúan las personas para absorber, procesar y generar información que se traduzca en decisiones que generen altos niveles de desempeño.

Las estructuras jerárquicas de control han sido dominantes en el entorno empresarial gracias, entre otras cosas, a las ventajas que ofrecen en la disminución de los costos de transacción [5]. Los costos de transacción hacen referencia al precio de negociar y renegociar los contratos o acuerdos.

La racionalidad limitada del ser humano, y por extensión el riesgo del oportunismo y del egoísmo en la toma de decisiones han presentado a las estructuras jerárquicas de control como un mecanismo más eficiente que el mercado para el desarrollo de intercambios caracterizados por la combinación de altos niveles de incertidumbre con el uso de activos específicos de propiedad establecida. Las jerarquías de control permiten monitorear los comportamientos individuales con el fin de disminuir la probabilidad de que el oportunismo y el egoísmo violen de manera explícita los acuerdos definidos.

Otro elemento que explica el uso de las estructuras jerárquicas de control en el contexto empresarial es la facilidad que brindan de vincular en una relación de dependencia a dos o más agentes (por ejemplo, la relación empleado-gerente), para disminuir la probabilidad de falla en las actividades delegadas (teoría de la agencia). Las fallas en las actividades delegadas se encuentran en estrecha relación con la racionalidad limitada del individuo y, puntalmente, con la información incompleta, imperfecta y asimétrica, así como con las dificultades que el agente pueda tener para procesar la información.

Pese a la predominancia de las estructuras jerárquicas de control en el contexto empresarial, diversas investigaciones han mostrado la precariedad de estas estructuras en el procesamiento deinformación. En una organización en la que cada actividad debe ser supervisada, coordinada y aprobada por una cadena formal de mando, existe una amplia probabilidad de que la cadena de mando quede saturada por las solicitudes de información y orientación de los subordinados. En las jerarquías puras, cada petición tiene que pasar por la cadena formal de mando antes de llegar al nodo antecesor común (ápice jerárquico). Para que la trasmisión de información sea exitosa depende de cada individuo que realiza esta actividad de procesamiento de información, pero no todos los individuos de la cadena de mando están igualmente cargados de responsabilidades: de hecho, algunos tienen mayor carga de actividades de producción; y otros, mayores cargas de procesamiento de información. Entre más arriba de la cadena de mando esté el individuo, más personas trasmitirán información a través de este y, por lo tanto, mayor es su carga de procesamiento.

A la fecha, se han planteado diferentes alternativas para superar las deficiencias de las estructuras jerárquicas de control. Una propuesta evidente es evitar el nodo o individuo sobrecargado creando un enlace adicional con otro nodo de la red que tenga una posición jerárquica similar. Sin embargo, establecer y mantener nuevos enlaces les quita a los individuos tiempo para las actividades de producción, de ahí que tanto la congestión como crear enlaces resulten costosos para el desempeño del sistema [6]. También se han planteado alternativas, como aplanar las estructuras, optimizar las actividades realizadas por los individuos [7], entre otras propuestas que permiten aumentar la eficacia, pero no generan cambios substanciales en el procesamiento de información.

Las empresas que fallan en el procesamiento de información tienen dificultades para resolver problemas y, por ende, son deficientes para afrontar la incertidumbre, el cambio, y de manera amplia no logran adaptarse a las condiciones dinámicas del entorno [6]. La alternativa para superar tal falla es comprender las organizaciones como sistemas procesadores de información, en las que la función de la estructura organizacional es gestionar de manera eficiente grandes volúmenes de información sin sobrecargar ninguno de los procesadores individuales.

A la fecha, las redes físicas que procesan información de manera altamente eficiente, por ejemplo, Internet o las redes sociales, se caracterizan por la ausencia de jerarquías de control. Estas redes no dependen de uno o varios controladores centrales (individuos con mejor posición jerárquica) para tomar decisiones, y los comportamientos surgen a partir de la interacción entre los nodos (individuos). 
El diseño de estructuras organizacionales diferentes a las estructuras jerárquicas de control se convierte en un desafío en el contexto empresarial, principalmente por la creencia que la ausencia de control jerárquico conduce al caos y la inestabilidad. A la fecha, son escasas las evidencias empíricas de estructuras organizacionales diferentes al control jerárquico que se gestiona en las empresas. Sin embargo, algunos de los acercamientos realizados al respecto muestran mayores niveles de agilidad en la toma de decisiones, creatividad, cooperación y lealtad, entre otras ventajas consideradas favorables para la adaptación de las organizaciones al entorno y, por extensión, para el logro de resultados empresariales.

\section{Materiales y métodos}

Para el desarrollo del documento, se realiza la revisión de literatura de investigaciones publicadas en el periodo 2016-2010, en los veinte principales journals de la categoría "Business, Management and Accounting" de Scimago, en la que se hace referencia a estructuras organizacionales flexibles, adaptativas y nuevas tendencias en gobernanza empresarial, principalmente. Dentro de los veinte principales journals la temática ha sido mayormente abordada en diez de ellos. En la tabla 1 se presenta la cantidad de artículos hallados en cada journal, así como su posición dentro del ranking. El journal que dentro del periodo de revisión lidera la temática es Organization Science con ocho artículos, seguido de Strategic Management Journal con siete artículos. En promedio, se encuentran alrededor de cuatro documentos por journal del tema tratado.

Tabla 1. Listado de los journals principales según Scimago con la cantidad de artículos encontrados de cada uno

\begin{tabular}{|c|l|c|}
\hline $\begin{array}{c}\text { Posición } \\
\text { en el } \\
\text { ranking }\end{array}$ & \multicolumn{1}{|c|}{ Journals } & $\begin{array}{c}\text { Cantidad } \\
\text { de artículos } \\
\text { en cada } \\
\text { journal }\end{array}$ \\
\hline 1 & Academy of Management Review & 5 \\
\hline 2 & $\begin{array}{l}\text { Academy of Management } \\
\text { Journal }\end{array}$ & 5 \\
\hline 3 & Organization Science & 8 \\
\hline 4 & Journal of Marketing & 0 \\
\hline 5 & $\begin{array}{l}\text { Journal of Operations Mana- } \\
\text { gement }\end{array}$ & 1 \\
\hline
\end{tabular}

\begin{tabular}{|c|c|c|}
\hline $\begin{array}{l}\text { Posición } \\
\text { en el } \\
\text { ranking }\end{array}$ & Journals & $\begin{array}{c}\text { Cantidad } \\
\text { de artículos } \\
\text { en cada } \\
\text { journal }\end{array}$ \\
\hline 6 & Journal of Management & 0 \\
\hline 7 & Personnel Psychology & 5 \\
\hline 8 & Strategic Management Journal & 7 \\
\hline 9 & $\begin{array}{l}\text { MIS Quarterly: Management } \\
\text { Information Systems }\end{array}$ & 0 \\
\hline 10 & International Organization & 0 \\
\hline 11 & Information and Organization & 0 \\
\hline 12 & $\begin{array}{l}\text { Organizational Research } \\
\text { Methods }\end{array}$ & 0 \\
\hline 13 & $\begin{array}{l}\text { Journal of Supply Chain Ma- } \\
\text { nagement }\end{array}$ & 5 \\
\hline 14 & Journal of Conflict Resolution & 0 \\
\hline 15 & Journal of Management Studies & 0 \\
\hline 16 & $\begin{array}{l}\text { Organizational Behavior and } \\
\text { Human Decision Processes }\end{array}$ & 4 \\
\hline 17 & $\begin{array}{l}\text { Management and Organization } \\
\text { Review }\end{array}$ & 0 \\
\hline 18 & Decision Support Systems & 2 \\
\hline 19 & Organization Studies & 0 \\
\hline 20 & $\begin{array}{l}\text { Academy of Management } \\
\text { Annals }\end{array}$ & 2 \\
\hline
\end{tabular}

Fuente: elaboración propia

Una vez seleccionados los artículos de cada uno de los journals investigados, se procedió a realizar un mapa conceptual que permitiera identificar la idea principal tratada en cada artículo, así como las características, ventajas y limitaciones de las estructuras organizacionales flexibles. En la parte final del documento se plantean las conclusiones y futuros trabajos de investigación en el diseño de estructuras organizacionales que incrementen la capacidad de adaptación del sistema a las condiciones cambiantes del entorno.

\section{La organización empresarial como sistema de procesamiento de información}

La ausencia de respuesta de las jerarquías de control a las condiciones cambiantes del entorno se encuentra en estrecha relación con los altos niveles de incertidumbre del entorno empresarial $y$, 
de manera puntual, con la complejidad creciente que caracteriza el entorno en el que actúan [8]. Una alternativa para superar la ausencia de respuesta de las jerarquías de control y aprovechar la complejidad creciente del entorno en la toma de decisiones empresariales es reconocer que los individuos, cuando resuelven problemas en entornos complejos, compensan su conocimiento limitado intercambiando información, conocimientos, experiencias, consejos, etc., con otros con los que interactúan de manera directa o indirecta [6]. Aprovechar la complejidad para tomar "buenas" decisiones empresariales requiere comunicación entre individuos dependientes en el sentido de que uno tiene la información que el otro necesita.

El problema de aprovechar la complejidad es equivalente al problema de la comunicación distribuida, abordado en la ciencia de redes desde el procesamiento de información. Si el entorno cambia rápidamente, los problemas empresariales también, y, por tanto, las empresas que se les dificulta la comunicación distribuida no logran resolver problemas y, en general, no logran adaptarse a las condiciones cambiantes del entorno.

La organización empresarial como sistema de procesamiento de información es una alternativa para superar el problema de la adaptación a las condiciones cambiantes. Esta alternativa plantea comprender la estructura organizacional como una red cuya función es gestionar grandes volúmenes de información de manera eficiente y sin sobrecargar ningún individuo del sistema. La eficiencia en el procesamiento de información se encuentra relacionada con buenas y ágiles decisiones que le permitan a las organizaciones empresariales modificar, modificarse y ajustarse al entorno en el que se encuentran. La forma como la organización solucione los problemas que el entorno necesita se encuentra influida por el tipo de estructura de red organizacional que adopte el sistema.

\section{Estructuras organizacionales y adaptabilidad}

A partir de la revisión de literatura, es posible identificar dos amplias alternativas, estrechamente relacionadas, en las que la flexibilización de las estructuras organizacionales ha sido abordada para promover la adaptabilidad de las organizaciones empresariales a las condiciones cambiantes del entorno. Por una parte, se encuentran las propuestas que enfocan la modificación de la estructura y, por otra, las propuestas que modifican la dinámica de la estructura jerárquica. Las primeras rediseñan la red de interacciones partir de criterios diversos como la descentralización del poder, las relaciones entre individuos por similitud o afinidad, los vínculos formales e informales, entre otros criterios, que le permitan a la organización absorber mayores niveles de información para la solución de problemas. El segundo grupo de propuestas enfoca la posibilidad de generar información (ideas, propuestas, novedades, alternativas de solución) a partir de facilitar la libre circulación de flujos de información en la organización.

\subsection{Propuestas enfocadas a la modificación de la estructura}

Dentro de las propuestas que enfocan la modificación de la estructura organizacional a fin de absorber mayores niveles de información para la solución de problemas, se encuentra el diseño de heterarquías [9]. En estas estructuras, el poder no está centralizado y circula entre los individuos según las circunstancias y necesidades del sistema. La rotación del poder depende de los recursos que proporcionen los individuos para resolver los problemas del entorno (conocimiento, experiencia, competencias, información, etc.). En [9], se muestra que el desequilibrio de poder incrementa los niveles de creatividad en el equipo y facilita la cooperación; sin embargo, la investigación también manifestó que estos resultados se encuentran relacionados con la percepción de legitimidad de quien asume el poder.

En la misma línea de la heterarquía, se encuentra la propuesta del liderazgo compartido o colaborativo de [10]. Desde esta perspectiva, el liderazgo es emergente y lo adquieren los individuos que logran destacarse de los demás por la forma como afrontan los desafíos del ambiente. El liderazgo compartido propone el surgimiento y coexistencia de diferentes líderes, que cambian en función de las condiciones de entorno, es decir, cualquier miembro de la organización, dependiendo de las situaciones, puede ser líder. El liderazgo compartido plantea como principal ventaja la posibilidad de tratar un mayor número de asuntos y, de manera amplia, problemas organizacionales que 
para un único individuo implicarían importantes esfuerzos de dirección y coordinación [10]. El liderazgo colaborativo desarrollado por Hernández parte de la premisa de que los individuos son más propensos a comportarse de manera colaborativa y beneficiar a los demás, aun sin tener en cuenta su propio bienestar cuando experimentan empatía. El liderazgo colaborativo sugiere el desarrollo de dinámicas compartidas que se fortalecen a través de la interacción entre las personas [11]-[13].

La estructura organizacional desde la heterarquía y el liderazgo compartido o colaborativo sugiere el desarrollo de un sistema relacional en el que el poder relativo entre los miembros del equipo se desplaza en el tiempo considerando los recursos relevantes. Lo anterior hace referencia a un poder existente, pero no constante, que depende de los recursos que las personas dispongan para hacer frente a las condiciones cambiantes del entorno.

La absorción de información del entorno en el diseño de estructuras organizacionales ha llevado al reconocimiento de las interacciones que los empleados tienen con personas externas y ajenas a la organización (redes externas), así como las interacciones que tienen con sus compañeros de trabajo (redes internas) [14]. Las redes externas facilitan el acceso a mayores fuentes de recursos (información, conocimiento, experiencia, capital, etc.); sin embargo, pueden generar mayor rotación de personal por la percepción de mejores oportunidades al tener los empleados relaciones externas, a las cuales pueden acudir en busca de recursos de información acerca de ofertas laborales o por la percepción de nuevas oportunidades [14]. Las redes internas pueden generar intercambios positivos y beneficiosos de los recursos interpersonales como la satisfacción laboral (el intercambio de los recursos aumenta la percepción de apoyo del compañero de trabajo) y el arraigo en el empleo (relacionado con el sentimiento de vinculación y gusto por el trabajo) [14], [15].

Algunas opciones de redes externas se encuentran en los agujeros estructurales, como han sido denominados por diversos autores [16] y [17]. Estas estructuras consisten principalmente en la aparición de fuentes de información externas a la organización y que se encuentran en interacción con algún miembro de la red. Los agujeros se asocian positivamente con la creatividad y la toma de decisiones, ya que amplían la pluralidad de percepciones frente a diferentes problemáticas; cada miembro del sistema es capaz de analizar y depurar la infor- mación obtenida y producir su propia lectura del entorno [16]-[19]. Adicionalmente, la existencia de múltiples fuentes de información permite el reconocimiento de mayor cantidad de variables a considerar en el proceso de toma de decisiones, y, en general, facilita construir más amplias y profundas interpretaciones del entorno. Tener mayor número de vínculos a través de fronteras organizativas se asocia con la adopción de estrategias menos normativas [17], la adquisición de las capacidades competitivas [18] y la innovación de productos [19].

En la misma línea de la propuesta de agujeros negros, se encuentra [20], que plantea el diseño de la red organizacional en interacción con otras redes organizacionales con las que se comparte información. La red EGO, como la denomina el autor, consiste establecer relaciones con colegas lejanos (sin importar que actúen en otra organización), sin necesidad de tener que pasar por cada uno de los nodos que lo anteceden. Estas redes permiten visualizar las personas con las que los empleados están en interacción, y que podrían colaborar, facilitar recursos de cualquier tipo o suministrar información acerca de cómo solucionar algún dilema. La gestión sobre esta estructura organizacional enfoca el aumento de la conectividad (mayores conexiones en la red) para así incrementar y facilitar la difusión de información, y con esto fortalecer la adquisición de recursos y, por extensión, de nuevos conocimientos [20].

La relevancia del diseño de estructuras organizacionales a partir de los planteamientos de la red EGO se encuentra en la identificación de patrones de comportamiento relacionados con la influencia que ejercen ciertos nodos (personas dentro de la red), y que permitan anticipar futuros esquemas de actuación. Los patrones de comportamiento surgen del análisis de tres rasgos de la red: intermediación, debilidad del EGO, y densidad de la red. La intermediación hace referencia a la identificación de actores que facilitan las transacciones o la interacción con nodos estratégicos. La debilidad enfoca la capacidad de la empresa de conectar subredes no conectadas o de conectarse de manera efectiva (llegar en pocos saltos a la persona estratégica) con otras estructuras organizacionales. Por su parte, la densidad hace referencia a la identificación de nodos con mayor número de conexiones o conexiones efectivas [20].

La importancia de las redes externas en el diseño de las estructuras organizacionales se ha 
extendido incluso al desarrollo de acuerdos de flexibilidad entre empresas [21]; esto permite configurar redes complejas que integren proveedores, clientes, distribuidores, entre otros [21]-[23]. El principal propósito de los acuerdos de flexibilidad entre empresas es gestionar la incertidumbre y minimizar los costos operativos a través del uso de diferentes herramientas que permitan incrementar la capacidad de procesamiento de información del sistema en general. Por ejemplo, la emisión de información para mejorar la coordinación dentro de la red, para hacer frente a los cambios de la demanda, alianzas, entre otros [24] y [25].

Por su parte, el trabajo desde la perspectiva de las redes internas ha llevado a reconocer la existencia y coexistencia de las redes formales e informales en las organizaciones empresariales [26]. Las redes formales son determinadas por la dirección de la organización y muestran las relaciones tanto de subordinación como de afiliación (departamental, divisional, etc.) entre los empleados del sistema; estas redes se basan en acuerdos institucionales (normas, estándares, protocolos, etc.) como medio para coordinar las actividades [27]. Las redes informales, por su parte, son emergentes y señalan los lazos sociales que existen en los empleados. Estas redes surgen de la similitud social y de la proximidad entre los individuos. La similitud se puede atribuir a los rasgos particularmente comunes, como la edad, ideales sociales, culturales, etc.; la proximidad se refiere a la cercanía y la frecuencia con la que los individuos interactúan por sus locaciones como la disposición de los puestos de trabajo, lugar de residencia, etc. [28].

Las ventajas y limitaciones de las redes formales e informales en el diseño de estructuras organizacionales han sido ampliamente abordadas en la literatura. Las redes formales son valoradas por el respaldo institucional que reciben. Al ser incentivadas por el núcleo administrativo reciben mayor apoyo para su gestión. Sin embargo, no garantizan la empatía entre las partes. Las redes informales, por su parte, fomentan la existencia de vínculos fuertes entre los empleados, los cuales refuerzan la comunicación [29] y [28]; la confianza y la generación de ideas creativas [30]-[32], [35]; promueven la innovación [36] y [38], y permiten que las organizaciones que operan en entornos altamente cambiantes respondan de manera flexible y dinámica [39] y [40]. Las amplias ventajas de las redes informales sugieren la necesidad de generar espacios de inte- racción entre la gente contratada de tal forma que se favorezca el surgimiento de afiliación por similitud y proximidad (capacitaciones vinculadas, comunidades en línea para la organización, programas de tutorías, eventos empresariales, proyectos multifuncionales, entre otros) [14], [28]. La principal restricción de las redes informales es la resistencia que presentan a los esfuerzos de la gestión [41].

El reconocimiento de la coexistencia de las redes formales e informales en el diseño de estructuras organizacionales genera amplios beneficios en el desempeño empresarial; sin embargo, los beneficios son mayores al combinar adecuadamente los dos tipos de red. Las redes semiformales, como han sido denominadas por Biancani, Mcfarland y Dahlander, sugieren aprovechar el respaldo institucional de las redes formales con los altos niveles de confianza, creatividad, innovación y flexibilidad que generan las redes informales [41].

Los altos niveles de desempeño que alcanzan las redes semiformales se encuentran relacionados con la capacidad de procesamiento de información que las caracteriza [42]. Los miembros de la red buscan señales de fuentes formales e informales con el fin de dar sentido a su situación actual; los anterior debido a que los comportamientos son en gran parte impulsados por las interpretaciones socialmente compartidas de eventos y acciones [42].

Las redes semiformales surgen por decisiones del núcleo central de la organización, pero la afiliación de los empleados es voluntaria. Existen amplias alternativas para hacer operativas las redes semiformales. Por ejemplo, en Nokia los ejecutivos crean una hoja de ruta estratégica anual, que es compartida con los empleados con el propósito de motivar su integración en torno a objetivos o proyectos particulares, y formar equipos estratégicos por proyecto. Algunos de los miembros son nombrados por los ejecutivos, mientras que otros se unen voluntariamente [43] y [44]. En el Programa de Asistencia de British Petroleum, un equipo que trabaja en un proyecto especializado puede invitar a miembros de otros equipos que trabajan en otros proyectos para solicitar asesoramiento o revisar sus metas y planes. Los compañeros invitados asisten voluntariamente; la asistencia no es obligatoria o asignada por el jefe o el equipo. Las personas asisten porque quieren compartir experiencia, conocimientos o aprender de lo que otros están haciendo [43], [45]. 


\subsection{Propuestas enfocadas a la modificación de la dinámica de la estructura jerárquica}

Las propuestas señaladas en este apartado enfocan la adaptabilidad de la organización a las condiciones cambiantes del entorno a través de dinámicas que facilitan la coordinación del sistema mediante la libre circulación de flujos de información en la organización. Los flujos abiertos de información parten de la premisa de la necesidad de descentralizar la toma de decisiones, y promueven la generación de ideas, alternativas y, de manera amplia, propuestas de acción o solución a los diferentes problemas que genera el entorno [46], [47].

La descentralización en la toma de decisiones implica que cualquier miembro de la organización puede realizar lecturas del entorno y plantear propuestas de solución que podrían ser adoptadas por la empresa, lo cual no significa que necesariamente todas las propuestas sean adoptadas. Es decir, la descentralización en la toma de decisiones reconoce que la propuesta de solución puede venir de cualquier miembro de la organización [48]. Los esfuerzos realizados al respecto en estructuras jerárquicas de control por lo general se ven truncados debido a las barreras en la comunicación que impone la estructura. Por su parte, los resultados obtenidos en organizaciones con estructuras flexibles, como los señalados en el apartado anterior, son altamente positivos, debido a que los desequilibrios de poder presentan impactos importantes en la creatividad del sistema [9], [49].

Es posible identificar tres amplias vías en que a la investigación en toma decisiones en estructuras flexibles y descentralizadas ha sido abordada. Por una parte, se encuentran los trabajos orientados a identificar los factores que permiten incrementar la creatividad en el sistema; por otra, están las investigaciones que buscan proponer alternativas para la selección de la propuesta que debería ser adoptada, y, finalmente, existen estudios que buscan comprender por qué se adopta una propuesta de acción y no otra.

Dentro de las propuestas que buscan incrementar la creatividad a partir de flujos de información que circulen libremente por la estructura, se encuentran los espacios compartidos y los espacios en común [50]-[52]. Estos espacios enfocan el ámbito social de las personas y buscan su interacción para el desarrollo de actividades en las que se comparte algún interés (aficiones compartidas, pasiones, ideas, clubes de aficionados, grupos de interés, encuentros, reuniones informales, talleres de pequeña escala, etc.). La particularidad de estos espacios es que promueven el surgimiento o fortalecimiento de relaciones informales que, como se mencionó anteriormente, promueven la cooperación, la creatividad, la lealtad, entre otras condiciones favorables para el desempeño empresarial. La principal restricción de los espacios compartidos y comunes es que la informalidad que los caracteriza, así como el tiempo limitado e infrecuente, puede llevar a que las ideas que surjan no trasciendan a una nueva práctica y queden como ideas que surgieron pero no llegaron a desarrollarse. La alternativa planteada en [50] para superar esta restricción es facilitar la construcción de significados compartidos a través de la repetición estable, ordenada y frecuente de los encuentros.

También se destaca la propuesta orientada al desarrollo de retos de trabajo planteado en [53]. Desde esta perspectiva, un estado de alerta continuo despierta la agilidad y la avidez de estar al tanto de las situaciones cambiantes del entorno. Un estado alejado del equilibrio no significa caos, sino un estado de cambio de las personas y la organización ante condiciones emergentes del entorno. Los retos son planteados por cualquier miembro de la organización y permiten ir visualizando a aquel que los plantea como líder de la misma [53]. Además, ayudan a tener un aprendizaje organizacional excepcional que hace parte de un aspecto importante para la adaptación al medio ambiente cambiante [54].

Uno de los principales desafíos del incremento en la cantidad de propuestas de solución en entornos descentralizados es el conflicto que surge por la contraposición de intereses y, en consecuencia, la dificultad para seleccionar una alternativa. La propuesta desarrollada por Song, Shi, Ma, \& Yang plantea un modelo matemático denominado modelo de opinión dinámica, que tiene como objetivo aumentar la velocidad de convergencia y disminuir el grado de fragmentación de la opinión, para disminuir los errores tanto de omisión como de concesión que se pueden presentar cuando se consideran varios puntos de vista [55]. La velocidad de convergencia hace referencia al tiempo que tardan en estar de acuerdo todos los integrantes de la organización; por su parte, el grado de fragmentación considera el número de grupos de opinión. El modelo considera dos factores sumamente importantes en una organización, independientemente de la estructura que esta maneje, los cuales son la tolerancia (diferencia entre 
opiniones individuales) y la influencia de los agentes, que por medio de la interacción de variables continuas y discretas son evaluados de manera conjunta haciendo evidente el funcionamiento positivo de este tipo de modelos al analizar las medidas de desempeño de este, como lo son la velocidad de consenso y el grado de fragmentación [55], [56].

Es importante señalar que, a la fecha, existe poca evidencia empírica de organizaciones empresariales que tomen decisiones en estructuras plenamente descentralizadas. Sin embargo, las escasas evidencias que existen permiten denotar los altos niveles de desempeño alcanzados por estas organizaciones. Al respecto, se encuentra el caso de la compañía de tomates Morning Star en California, caracterizada por que "nadie es jefe de nadie”. En esta compañía, las decisiones se toman en comités creados y conformados de manera voluntaria. En la empresa cualquier persona puede tomar decisiones, incluso financieras; la única condición es que se haya consultado con expertos que emergen en la organización, que ganan reputación en la medida en que resultan exitosas las decisiones en las que participan [18]. También se destaca el caso de la compañía holandesa de servicios de enfermería domiciliarios Buurtzorg. Esta compañía opera en esquemas completamente descentralizados, incluso para la facturación (cada enfermera elabora sus propias facturas); sin embargo, según McEvily y Zaheer, presenta los mayores niveles de satisfacción de los empleados y la menor estructura de costos en comparación con otras firmas holandesas [18].
La escasa acogida que las organizaciones han presentado frente a la implementación de estructuras organizacionales diferentes a las jerarquías de control o que busquen modificar la dinámica de la estructura a través de la libre circulación de flujos de información, pese a los resultados positivos para la adaptación de la organización a las condiciones cambiantes del entorno, se encuentra relacionada con el supuesto, fuertemente arraigado en el paradigma dominante, de que la ausencia de control jerárquico o centralizado conduce al caos y que hay altos costos (tiempo, recursos) en la implementación de estas propuestas [57], [49]. Sin embargo, el fracaso empresarial debido a la poca adaptabilidad de las organizaciones a las condiciones cambiantes del entorno exige nuevas alternativas para la comprensión y diseño de las estructuras organizacionales.

\section{Principales características, ventajas y limitaciones de las estructuras organizacionales alternativas al control jerárquico}

A partir de la revisión bibliográfica realizada, se han identificado las características, ventajas y principales limitaciones que tienen las estructuras organizacionales alternativas al control jerárquico, y que promueven la adaptabilidad de la organización a las condiciones cambiantes del entorno (figura 1). Las características (señaladas por las

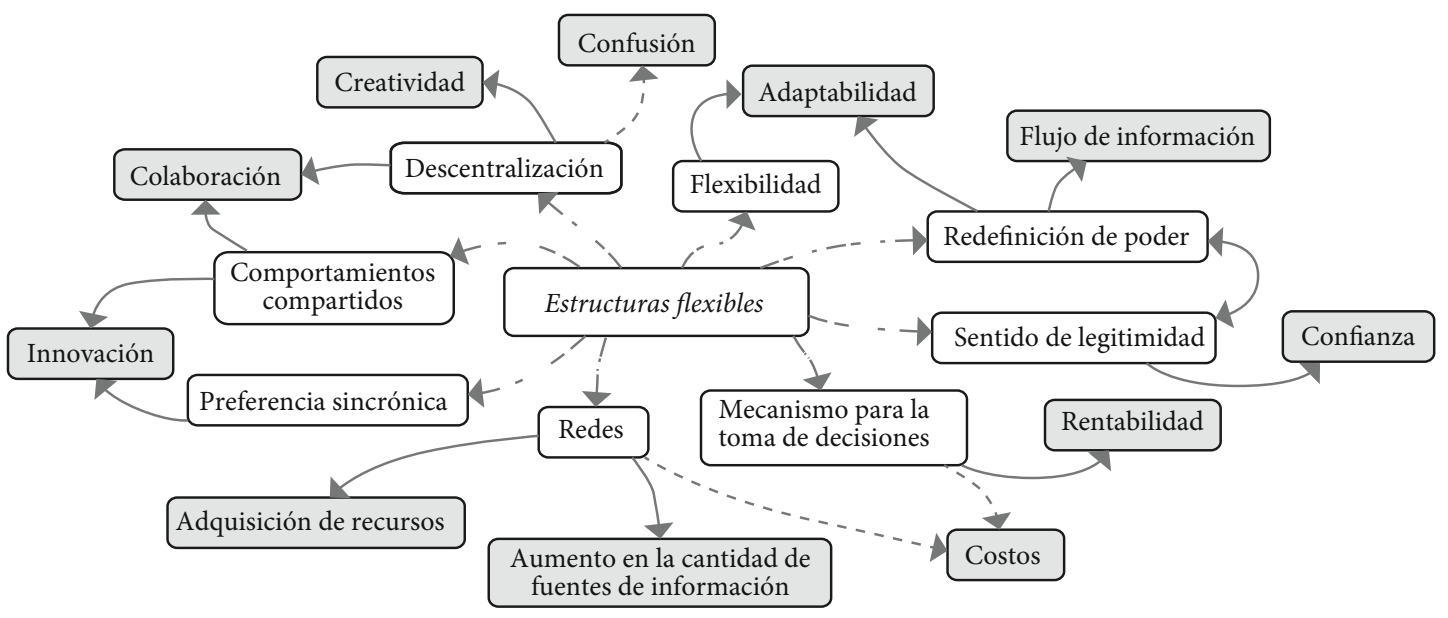

Figura 1. Mapa relacional de características, limitaciones y ventajas de las estructuras flexibles Fuente: elaboración propia 
flechas semipunteadas) hacen referencia a los rasgos estructurales y de dinámica que predominan en las estructuras flexibles estudiadas; las ventajas (señaladas por las flechas continuas) reúnen los principales beneficios que estas estructuras tienen en el desempeño empresarial, y las limitaciones (sombreadas en color verde) hacen referencia a las dificultades y, mejor aún, a los retos que la implementación de esas estructuras pueden acarrear.

\subsection{Características de las estructuras organizacionales flexibles}

Dentro de las características identificadas se encuentran la descentralización de la información, la modificación en la definición de poder, la facilidad de interacción entre las partes, el sentido de legitimidad del poder, la existencia de mecanismos para la toma de decisiones, el conocimiento compartido, los comportamientos compartidos y la preferencia sincrónica. Estas características, tanto de manera individual como en conjunto, pueden proporcionar una serie de ventajas competitivas para las organizaciones, que facilitan la adaptación de las organizaciones a las condiciones cambiantes del entorno.

La descentralización de la información puede considerarse como la característica más importante en este tipo de estructuras, principalmente por la facilidad que bridan para el procesamiento de información. La descentralización disminuye la sobrecarga de información en los nodos, lo que permite reducir la distorsión de información y los tiempos para la toma de decisiones, aunque también aumenta la probabilidad de ocurrencia de errores de omisión de forma intencional o no intencional [58], [59].

Una característica derivada de la descentralización es el cambio en la posesión del poder. En las organizaciones flexibles, la rotación del poder y poder distribuido en todo el sistema son algunas de las alternativas para disminuir la sobrecarga y distorsión de la información, y agilizar la toma de decisiones [9].

La facilidad de interacción entre los individuos incrementa tanto la probabilidad de flujos de información que circulan por toda la organización como la capacidad de procesamiento, lo cual proporciona decisiones oportunas frente a las circunstancias cambiantes del entorno. La facilidad de interacción aprovecha las ventajas de la comunicación formal e informal entre las partes tanto a nivel externo como interno [60], [61].
El sentido de legitimidad de poder hace referencia al reconocimiento que la organización hace frente a la influencia social que uno o varios individuos pueden generar en un momento determinado. Las estructuras flexibles plantean la división del poder en formas distintas y cambiantes en el tiempo (heterarquías, liderazgo compartido, etc.); el poder es rotativo y, por tanto, todos los individuos pueden tener igual de importancia en la toma de decisiones. La legitimidad del poder permite que la dinámica que maneja la organización se mantenga y genere el rendimiento deseado [59], [12].

La implementación de mecanismos para la toma de decisiones permite aumentar la velocidad con la que las decisiones surgen en la organización y promueve mayor aprovechamiento de los recursos; es importante señalar que estos mecanismos no generan necesariamente una convergencia de las partes hacia una decisión, sino que pueden optar por alternativas que promuevan el disenso [55], [56].

El conocimiento compartido se alcanza cuando los individuos tienen información similar frente a diferentes circunstancias del entorno e incluso podrían, aunque no necesariamente, desarollar una misma interpretación de una situación. Este conocimiento disminuye la probabilidad de falla en el sistema, pues todos los individuos disponen de información relevante para la toma de decisiones, lo que incrementa la robustez para desempeñarse en ambientes complejos y se manifiesta en agilidad en la respuesta. El conocimiento compartido se logra en la medida en que las partes interactúan y comparten información. Es importante señalar que, en las organizaciones flexibles, este no es un objetivo en sí mismo, sino el resultado de la interacción entre las partes [61].

El conocimiento compartido facilita el desarrollo de comportamientos colectivos, entendidos como las acciones sincronizadas desarrolladas por dos o más individuos. Los comportamientos colectivos no implican el consenso de los miembros de la organización y facilitan comprender la emergencia de decisiones empresariales. Los comportamientos colectivos surgen de la disposición de cada individuo para adaptarse al ritmo del sistema con el propósito de crear sincronía con los demás, más específicamente con aquellos con quienes interactúa y comparte información. Una preferencia sincrónica alta implica una disposición a renunciar al control sobre el ritmo de las actividades propias, 
para adaptarse a las de los demás, con tal de culminar con éxito la tarea [62].

Las características de las estructuras flexibles incrementan la capacidad del sistema de enfrentar cambios inesperados y de proponer alternativas para adaptarse ágilmente a las condiciones cambiantes del entorno [12].

\subsection{Ventajas de las estructuras organizaciones flexibles}

La revisión de literatura permite identificar diferentes ventajas que desarrollan las organizaciones empresariales caracterizadas por estructuras organizacionales flexibles. La principal, sin lugar a dudas, es el incremento de la capacidad de adaptación a las condiciones cambiantes del entorno, que se encuentra en estrecha relación con otras ventajas alcanzadas, como mayores niveles de innovación, creatividad, incremento de la confianza entre las partes, colaboración, arraigo en la organización, mejor aprovechamiento de los recursos, desarrollo de alianzas, coordinación, flujos de información eficientes y agilidad en la toma de decisiones [63].

La adaptación a las condiciones cambiantes del entorno refleja la capacidad de la organización de modificar, cambiarse y ajustarse de manera ágil a los cambios que le exige el ambiente en el que actúa. La investigación desarrollada por [21] mostró que la descentralización y, de manera amplia, la flexibilidad para la toma decisiones incrementan los niveles de adaptación organizacional. La capacidad de adaptación facilita la identificación y acceso a recursos internos y externos, que pueden ser combinados y recombinados para obtener beneficios para la organización [21]-[23].

La adaptabilidad se encuentra en estrecha relación con la creatividad y la innovación. Las estructuras flexibles promueven la creatividad por medio de la renovación de los vínculos entre las personas, lo que facilita el reconocimiento y la generación de nuevas formas de pensar, nuevos modelos mentales $y$, de manera amplia, el surgimiento de ideas, alternativas y propuestas de acción diferentes [30]. Crear una gran y enriquecedora fuente de creatividad da paso a nuevas ideas y futuras prácticas innovadoras que surgen como respuesta de los cambios del entorno y permitirían aumentar el desempeño laboral.

En esta línea se encuentra el trabajo desarrollado por [51]. En el primero, realizado en el departamento de desarrollo de software de una empresa europea, se expone el aumento de las ideas creativas en la medida en que se fortalece y se renueva la interacción entre las partes. Los espacios compartidos, al igual que la inclusión de nuevos empleados en los equipos de desarrollo, se constituyen como mecanismos que promueven la creatividad por el aumento en los flujos de información que circulan en el sistema. El trabajo de Furnari [51] presenta, entre otros, el caso del Homebrew Computer Club (HCC) el cual marca el inicio de prácticas innovadoras y de alto impacto en la industria de la computación. HCC inicia lo que a la fecha se conoce como clubes de informáticas, los cuales promueven los encuentros informales entre personas interesadas en ciertos temas en común.

La investigación realizada por S. Carnovale y S. Yeniyurt [20] muestra que el fortalecimiento de la interacción entre las partes en la estructura organizacional (aumento de la densidad de la red) incrementa la conectividad, y con esta hay más recursos disponibles para facilitar la difusión de conocimientos. Según el autor, estos podrían tener impacto en la innovación, el desarrollo de alianzas, la coordinación entre las partes y la toma de decisiones.

De acuerdo con [14], las redes organizacionales caracterizadas por fuertes niveles de interacción favorecen el deseo de los empleados de permanecer con sus empleadores, lo que genera intercambios positivos y beneficiosos de los recursos interpersonales, como la satisfacción laboral y el arraigo por el empleo. Adicionalmente, también pueden influir tanto en el fortalecimiento de la colaboración, entendida como la tendencia a ayudar y a crear un vínculo en donde las personas se sienten satisfechas con su trabajo y compañeros, como en el intercambio de información; esto se traduce en el desarrollo de ventajas empresariales [14].

Las redes organizacionales marcadas por fuertes niveles de interacción y flexibilidad para la comunicación favorecen la apertura hacia ambientes de confianza y la construcción de lazos de amistad [63]. Los aspectos positivos de tener amistades laborales que también son amigos cercanos son que hay un gran apoyo emocional y una colaboración amistosa que se brinda en el ambiente laboral y no laboral. Desde la perspectiva laboral, la confianza y la amistad entre las partes favorecen la creación de fuentes de recursos para la solución de problemas. Cuando un empleado requiere información adicional para el desarrollo del trabajo, usualmente pide 
consejo o explicación a las personas que considera cercanas [64].

La confianza y colaboración puede extenderse de las personas a las empresas, y con esto configurar el surgimiento de alianzas o asociaciones. Empresas ajenas se pueden conectar con otras por medio de intermediarios, así como saltar a aquellos huecos o espacios sin conexión. La ventaja competitiva surge de agujeros estructurales en los que el intermediador puede manejar la información lejana a través de grupos [20]. En las subredes se trata un patrón de comportamiento importante, que es la debilidad de la red. Aparentemente, la palabra debilidad puede parecer un aspecto negativo. Pero, en este caso, es todo lo contrario, por lo ya mencionado anteriormente. Contener redes débiles hace que no se sature de información los nodos, sino que la información viaje directamente a su destino final [20]. Todo esto unifica el resultado al que se pretende llegar: un flujo de información eficiente que da paso a la buena toma de decisiones.

\subsection{Limitaciones de las estructuras organizacionales flexibles}

Dentro de las principales limitaciones de las estructuras flexibles, se encuentran el aumento de las fuentes de información, lo que incrementa la complejidad del sistema para la toma de decisiones, así como los costos que acarrea la incorporación de tecnologías de la información para facilitar la interacción entre las partes.

La descentralización, a pesar de que aumenta la capacidad de adaptación al cambio para la organización, puede traer consigo una complicación con el proceso de información, dado que la existencia de diversas fuentes de información puede generar confusión por parte de los miembros de la red [65], [48].

La complejidad de la red se ve envuelta en aspectos de diversidad debido a que las fuentes de información se incrementan, y esto produce un cierto desconcierto entre los integrantes de la red, ya que puede existir información contraria proveniente de dos o más fuentes diferentes con el mismo nivel de certeza. Para tal manejo de información, se requieren herramientas informáticas que ayuden a acelerar los procesos para sintetizarla y den una visión más objetiva de los datos; así, las decisiones podrán verse desde enfoques más globales y objetivos de los logros importantes que requiere la organización.
Así mismo, se deben entablar nuevos lazos, lo cual requiere un mantenimiento constante de las redes desde aspectos como su estudio hasta su prevalencia en el tiempo, además de la implementación de mecanismos de tomas de decisiones y la utilización de herramientas como TIC para proporcionar una red segura y estable para el flujo de información. Como se ilustra en la figura 1, todo esto puede acarrear costos considerables para la organización, pero nunca superaran a todas las ganancias que se generaran con estos cambios, pero todo esto debe verse a largo plazo. De todas formas, un cambio en la estructura organizacional o en los factores que afectan la dinámica organizacional siempre requerirá de una inversión inicial y continua, que debe ser evaluada por la organización para considerar si es efectivo y viable realizar dichos cambios [66].

\section{Discusión}

El paradigma dominante hasta la fecha son las estructuras jerárquicas de control y ha sido así década tras década. Estas establecen patrones de comunicación en torno al estructurado poder. Sin embargo, como hemos podido establecer, estas estructuras pudieron ser necesarias en épocas pasadas cuando no había globalización y no existía una demografía tan extensa, dado que, aunque estamos en una era de consumismo, ahora se presenta una mayor oferta que demanda, las empresas de una u otra forma producen tantos productos que no hay suficientes compradores. En este sentido, muchas empresas pueden fracasar: existen aterradoras estadísticas alrededor de ello, por lo que la metodología de procesamiento de la información (base fundamental en el manejo de un sistema cualquiera) debe cambiar rotundamente, tal como ha cambiado marcadamente el mundo estos últimos años gracias a la tecnología.

Lo que se propone aquí nos brinda no solo una alternativa efectiva, sino una innumerable cantidad de estas para poder enfrentar tal entorno cambiante. La flexibilidad en las organizaciones puede efectuarse drásticamente en un cambio en la estructura $\mathrm{o}$, menos drásticamente, en su dinámica. Dentro de estas dos ramas explicadas anteriormente, se tienen diversas opciones, que se pueden aplicar a cualquier contexto organizacional. 
Las opciones expuestas con anterioridad como las redes, los mecanismos para la toma de decisión, el sentido de legitimidad, la redefinición del poder, la flexibilidad, la descentralización, los comportamientos compartidos y la preferencia sincrónica no solo llevan a lo que antes buscaban las organizaciones, sostenibilidad, crecimiento y rentabilidad, que no son garantía fiable del éxito de una organización, sino que además aportan creatividad, colaboración, innovación, adquisición de todo tipo de recursos, mayor cantidad de fuentes de información, confianza, sin dejar de lado la confusión y los costos, para finalmente llegar a la adaptabilidad. Así se puede tener un flujo de información efectiva, que podrá dar a conocer un panorama más amplio del entorno, la competencia y las tendencias, entre otros; de esta manera, será posible tomar las mejores decisiones para el éxito de la compañía.

La importancia que se destaca en el artículo es la necesidad de generar un impresionante cambio que genere, así mismo, un impactante viraje en las organizaciones. Este lleva consigo el tomar las mejores decisiones a través de la absorción de la mayor cantidad posible de información efectiva y el procesamiento de esta sin pérdida de datos importantes en su flujo, lo que lleva finalmente a la adaptabilidad. Las diferentes fuentes referenciadas dan cuenta de las innumerables situaciones en que se establece la superioridad del mensaje que se pretende transmitir, así como de estructuras que florecen por encima de aquellas que siempre florecieron con el anticuado paradigma dominante.

\section{Conclusiones y futuras líneas de investigación}

Las estructuras organizaciones flexibles constituyen una alternativa distinta a las estructuras jerárquicas de control. A la fecha existe poca evidencia empírica acerca del uso de estas estructuras en las organizaciones empresariales; sin embargo, los resultados presentados exaltan la capacidad de adaptación a las condiciones cambiantes del entono.

La capacidad de adaptación de las estructuras flexibles se encuentra en estrecha relación con el aumento en el procesamiento de grandes volúmenes de información. Lo anterior parte de la premisa que las condiciones cambiantes del entorno, así como la cada vez mayor facilidad de interacción entre las personas, generan nueva información que debe ser procesada por las organizaciones de manera ágil y oportuna, como condición necesaria para mejorar el desempeño del sistema.

La presente investigación permitió identificar las características, ventajas y limitaciones de las estructuras organizacionales flexibles, aspecto que a la fecha no había sido ampliamente abordado, principalmente por la predominancia de las estructuras jerárquicas de control. No obstante, la existencia de literatura que argumente la relevancia de la estructura organizacional en la adaptabilidad empresarial pone de manifiesto la relevancia de la temática, y abre la posibilidad a futuras investigaciones que permitan corroborar el impacto de la estructura, en particular, de las características señaladas en la presente investigación en la adaptabilidad del sistema y, de manera amplia, en el éxito empresarial. La aplicación directa de los posibles cambios a las estructuras organizacionales, señalada a lo largo de este artículo, permitiría el desarrollo de nuevas investigaciones que darían paso a un desarrollo óptimo de la temática, dado que la simulación de conductas de comportamiento de los individuos en la actualidad proporciona información sesgada por aquellas variables que no pueden ser simuladas de una forma acertada en su totalidad. Pero, sin duda, es un inicio y avance clave, por lo que para futuras investigaciones se propone parametrizar las características del paradigma dominante frente a lo propuesto en el presente trabajo. Con ello se podría modelar el comportamiento organizacional bajo condiciones cambiantes del entorno. Teniendo un modelo fiable a seguir a través de una modelación, es probable que este se pueda aplicar sin dificultad a una organización.

\section{Referencias}

[1] P. BeomCheol Kim, S. Kimb y S. Seongs, "Organizational Drivers and Outcomes of Casino Employees", J. Hosp. Mark. Manag, pp. 2-21, 2016. [Online]. Disponible en https://www.cabdirect.org/cabdirect/ abstract/20173147288

[2] M. Sigala y O. Kyriakidou, "Creativity and innovation in the service sector", Serv. Ind. J., vol. 35, pp. 297-302, 2015. [Online]. doi: https://doi.org/10.108 0/02642069.2015.1010159

[3] E. D. Pulakos, S. Arad, M. A. Donovan y K. E. Plamondon, "Adaptability in the workplace: Development of a 
taxonomy of adaptive performance", J. Appl. Psychol., vol. 85, pp. 612-624, 2000. [Online]. http://psycnet. apa.org/journals/apl/85/4/612/

[4] N. Siggelkow y D. A. Levinthal, “Temporarily Divide to Conquer: Centralized, Decentralized, and Reintegrated Organizational Approaches to Exploration and Adaptation", Organ. Sci., vol. 14, n. ${ }^{\circ} 6$, pp. 650669, 2003. [Online]. doi: http://doi.org/10.1287/ orsc. 14.6.650.24840

[5] O. Williamson, Markets and Hierarchies: Analysis and Antitrust Implications, Nueva York: The Free Press, 1975. [Online]. Disponible en http://garfield.library. upenn.edu/classics1988/A1988M191900001.pdf

[6] D. J. Watts, Seis grados de separación. La ciencia de las redes en la era del acceso, Barcelona: Paidós, 2006. [Online]. Disponible en https://books. google.com.co/books?id=jt4ktVJ427QC\&print$\mathrm{sec}=$ frontcover $\& \mathrm{hl}=\mathrm{es} \&$ source $=$ gbs_ge_summary_r\&cad $=0 \# \mathrm{v}=$ onepage $\& \mathrm{q} \& \mathrm{f}=$ false

[7] I. Hofacker y R. Vetschera, "Algorithmical approaches to business process design", Comput. Oper. Res., vol. 28, n. ${ }^{\circ}$ 13, pp. 1253-1275, 2001. [Online]. Disponible en doi: https://doi.org/10.1016/S03050548(00)00038-1

[8] L. E. Bohórquez, "La comprensión de las organizaciones empresariales y su ambiente como sistemas de complejidad creciente: rasgos e implicaciones", Rev. Ing., vol. 21, n. ${ }^{\circ}$, pp. 363-377, 2016. [Online]. doi: https://doi.org/10.14483/udistrital.jour. reving.2016.3.a07

[9] F. Aime, S. Humphrey, D. S. Derue y J. B. Paul, “The riddle of heterarchy: Power transitions in crossfunctional teams", Acad. Manag. J., vol. 57, n. ${ }^{\circ}$ 2, pp. 327352, 2014. [Online]. doi: https://doi.org/10.5465/ amj.2011.0756

[10] B. R. Spisak, M. J. O’Brien, N. Nicholson y M. Van Vugt, "Niche construction and the evolution of leadership", Acad. Manag. Rev., vol. 40, n. ${ }^{\circ}$ 2, pp. 291306, 2015. [Online]. doi: https://doi.org/10.5465/ amj.2011.0756

[11] M. Hernández, "Toward an Understanding of the Psychology of Stewardship", The Academy of Management Review, vol. 37, n. ${ }^{\circ}$ 2, pp. 172193, 2012. [Online]. doi: https://doi.org/10.5465/ amr.2010.0363

[12] L. White, G. Currie y A. Lockett, "Pluralized leadership in complex organizations: Exploring the cross network effects between formal and informal leadership relations", Leadersh. Q., vol. 27, n. 2, pp. 280 297, 2016. [Online]. Disponible en doi: https://doi. org/10.1016/j.leaqua.2016.01.004

[13] D. E. Chandler, K. E. Kram y J. Yip, "An Ecological Systems Perspective on Mentoring at Work: A Review and Future Prospects", Acad. Manag. Ann., vol. 5, n. ${ }^{\circ}$ 1, pp. 519-570, 2011. [Online]. doi: https://doi.org/10 $.1080 / 02642069.2015 .1010159$

[14] C. M. Porter, S. E. Woo y M. A. Campion, "Internal and External Networking Differentially Predict Turnover Through Job Embeddedness and Job Offers", Pers. Psychol., n. ${ }^{\circ}$ 69, pp. 635-672, 2016. [Online]. doi: https://doi.org/10.1111/peps.12121

[15] J. Battilana, "Change Agents, Networks, and Institutions: A Contingency Theory of Organizational Change", Acad. Manag. J., vol. 55, n. ${ }^{\circ}$ 2, pp. 381 398, 2012. [Online]. doi: https://doi.org/10.5465/ amj.2009.0891

[16] X. Zou y P. Ingram, "Bonds and boundaries: Network structure, organizational boundaries, and job performance", Organ. Behav. Hum. Decis. Process, vol. 120, n. ${ }^{\circ} 1$, pp. 98-109, 2013. [Online]. doi: https://doi.org/10.1016/j.obhdp.2012.09.002

[17] M. A. Geletkanycz, "The salience of 'culture's consequences': The effects of cultural values on top executive commitment to the status quo", Strateg. Manag.

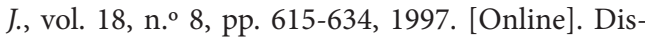
ponible en http://www.jstor.org/stable/3088179?seq=1\#page_scan_tab_contents

[18] B. McEvily y A. Zaheer, "Bridging ties: a source of firm heterogeneity in competitive capabilities", Strateg. Manag. J., vol. 20, n. ${ }^{\circ} 12$, pp. 1133-1156, 1999. [Online]. Disponible en http://www.jstor.org/ stable/3094082?seq=1\#page_scan_tab_contents

[19] J. A. Nickerson y T. R. Zenger, "A Knowledge Based Theory of the Firm: The Problem Solving Perspective", Organ. Sci., vol. 15, n. ${ }^{\circ}$ 6, pp. 617632, 2004. [Online]. doi: https://doi.org/10.1287/ orsc. 1040.0093

[20] S. Carnovale y S. Yeniyurt, "The role of ego network structure in facilitating ego network innovations", J. Supply Chain Manag., vol. 51, n. ${ }^{\circ}$ 2, pp. 22-46, 2015. [Online]. doi: https://doi.org/10.1111/jscm.12075

[21] L. C. V. Cheng, D. E. Cantor, C. M. Grimm y M. E. Dresner, "Supply Chain Drivers of Organizational Flexibility: A Study of U.s. Manufacturing Industries", J. Supply Chain Manag., vol. 50, n. ${ }^{\circ}$ 4, pp. 62-75, 2014. [Online]. doi: https://doi.org/10.1111/ jscm. 12075

[22] S. Brion y C. Anderson, "The loss of power: How illusions of alliance contribute to powerholders' downfall”, Organ. Behav. Hum. Decis. Process, vol. 121, n. ${ }^{\circ}$ 1, pp. 129-139, 2013. [Online]. doi: https:// doi.org/10.1016/j.obhdp.2013.01.005

[23] N. J. Foss y N. J. Foss, "Why a central network position isn't enough : sharing in employee networks", Acad. Manag. J., vol. 54, pp. 1277-1297, 2011. [Online]. doi: https://doi.org/10.5465/amj.2009.0007 
[24] C. Schmoltzi y C. M. Wallenburg, "Operational Governance in Horizontal Cooperations of Logistics Service Providers: Performance Effects and the Moderating Role of Cooperation Complexity", J. Supply Chain Manag., vol. 48, n. ${ }^{\circ}$ 2, pp. 53-74, 2012 [Online]. doi: https://doi.org/10.1111/j.1745493X.2011.03262.x

[25] R. Gulati, F. Wohlgezogen y P. Zhelyazkov, “The Two Facets of Collaboration: Cooperation and Coordination in Strategic Alliances", Acad. Manag. Ann., vol. 6 , n. ${ }^{\circ} 1$, pp. 531-583, 2012. [Online]. doi: https:// doi.org/10.5465/19416520.2012.691646

[26] C. Oswick, P. Fleming y G. Hanlon, "From borrowing to blending: Rethinking the processes of organizational theory building", Acad. Manag. Rev., vol. 36, n. ${ }^{\circ}$ 2, pp. 318-337, 2011. [Online]. doi: https://doi. org/10.5465/amr.2009.0155

[27] E. M. Tachizawa y C. Y. Wong, "The Performance of Green Supply Chain Management Governance Mechanisms: A Supply Network and Complexity Perspective", J. Supply Chain Manag., vol. 51, n. ${ }^{\circ}$ 3, pp. 18-32, 2015. [Online]. doi: https://doi. org/10.1111/jscm. 12072

[28] R. Reagans, "Close Encounters: Analyzing How Social Similarity and Propinquity Contribute to Strong Network Connections", Organ. Sci., vol. 22, March 2014, pp. 835-849, 2011. [Online]. doi: https://doi. org/10.1287/orsc. 1100.0587

[29] E. R. Crawford y J. A. Lepine, "A Configural Theory of Team Processes: Accounting for the Structure of Taskwork and Teamwork", Acad. Manag. Rev., vol. 38, n. ${ }^{\circ} 1$, pp. 32-48, 2013. [Online]. doi: https://doi. org/10.5465/amr.2011.0206

[30] M. E. Sosa, "Where Do Creative Interactions Come From? The Role of Tie Content and Social Networks", Organ. Sci., vol. 22, n. ${ }^{\circ}$ 1, pp. 1-21, 2011. [Online]. doi: https://doi.org/10.1287/orsc.1090.0519

[31] Y. H. Kim, F. J. Sting y C. H. Loch, "Top-down, bottom-up, or both? Toward an integrative perspective on operations strategy formation", J. Oper. Manag., vol. 32, n. ${ }^{\circ} 7-8$, pp. 462-474, 2014. [Online]. doi: https://doi.org/10.1016/j.jom.2014.09.005

[32] J. Brown y P. Duguid, "Organizational learning and communities of practice: Toward a unified view of working, learning, and innovation", Organ. Sci., vol. 2, n. ${ }^{0} 1$, pp. 40-57, 1991. [Online]. doi: https://doi. org/10.1287/orsc.2.1.40

[33] W. Powell, K. Koput y L. Smith Doerr, "Interorganizational collaboration and the locus of innovation: Networks of learning in biotechnology", Adm. Sci. Q., vol. 41, n. ${ }^{\circ}$ 1, pp. 116-145, 1996. [Online]. doi: https://doi.org/10.2307/2393988
[34] B. Uzzi y J. Spiro, "Collaboration and creativity: The small world problem", Am. J. Sociol., vol. 111, n. ${ }^{\circ}$ 2, pp. 447-504, 2005. [Online]. doi: https://doi. org $/ 10.1086 / 432782$

[35] Anne Bøllingtoft, Lex Donaldson, George P. Huber, Dorthe Døjbak Håkonsson, Charles C. Snow, Collaborative Communities of Firms. Purpose, Process, and Design, Springer Science \& Business Media, Londres, 2011.

[36] M. Aiken, S. Bacharach y J. French, "Organizational structure, work process, and proposal making in administrative bureaucracies", Acad. Manag. J., vol. 23, n. ${ }^{\circ}$ 4, pp. 631-652, 1980. [Online]. doi: https://doi. org $/ 10.5465 / 255553$

[37] M. Tushman, "Special boundary roles in the innovation process", Adm. Sci. Q., vol. 22, n. ${ }^{\circ} 4$, pp. 587-605, 1977. [Online].doi:https://doi.org/10.2307/2392402

[38] L. Fleming, S. Mingo, and D. Chen, "Collaborative brokerage, generative creativity, and creative success", Adm. Sci. Q., vol. 52, n. ${ }^{\circ}$ 3, pp. 443-475, 2007. [Online]. doi: https://doi.org/10.2189/asqu.52.3.443

[39] T. Burns y G. M. Stalker, "The Management of Innovation”, Econ. J., vol. 79, pp. 403-405, 1969. [Online]. Disponible en https://papers.ssrn.com/sol3/papers. cfm?abstract_id $=1496187$

[40] P. Lawrence y J. Lorsch, "Differentiation and Integration in Complex Organizations", Adm. Sci. Q., vol. 12, pp. 1-30, 1967. [Online]. doi: https://doi. org/10.2307/2391211

[41] S. Biancani, D. A. Mcfarland y L. Dahlander, "The Semiformal Organization", Organ. Sci. Artic. Adv., vol. 25, En. 2015, pp. 1-19, 2014. [Online]. Disponible en http://pubsonline.informs.org/doi/ abs/10.1287/orsc.2013.0882

[42] L.Festinger, "Atheoryofsocialcomparison processes", Human Relations, vol. 7, pp. 117-140, 1954. [Online]. doi: https://doi.org/10.1177/001872675400700202

[43] L. Gratton, "Four Ways to Encourage More Productive Teamwork", Harv. Bus. Rev., 2007. [Online]. Disponible en https://questionnaire.app.co.id/portal. nsf/37fab4341f7d072b4725704900075689/87849ff09c306c414725738a00085061/\$FILE/Harvard\%20 Management\%20Update\%202007-11.pdf

[44] L. Gratton, Hot Spots: Why Some Teams, Workplaces, and Organizations Buzz with Energy And Others Don't, Read How You Want, California, 2007.

[45] N. Dixon, Common Knowledge: How Companies Thrive by Sharing What They Know, Cambridge, MA: Harvard Bus. Sch. Press., 2000.

[46] H. Laihonen, "Knowledge flows in selforganizing processes", J. Knowl. Manag. vol. 10, n. ${ }^{\circ} 4$, pp. 127-135, 2006. [Online]. doi: https://doi. org/10.1108/13673270610679417 
[47] D. S. Alberts and R. E. Hayes, Power to the edge: Command, control in the information age, Washington: CCRP from Business Source Complete Database, 2003. [Online]. Disponible en http://www. dodccrp.org/files/Alberts_Power.pdf

[48] E. M. Wong, M. E. Ormiston y P. E. Tetlock, "The Effects of Top Management Team Integrative Complexity and Decentralized Decision Making on Corporate Social Performance Complexity on Corporate Social Performance", Acad. Manag. J., vol. 54, n. ${ }^{\circ}$ 6, pp. 1207-1228, 2011. [Online]. doi: https://doi. org/10.5465/amj.2008.0762

[49] Z. J. Zhao y J. Anand, "Beyond boundary spanners: the 'collective bridge' as an efficient interunit structure for transferring collective knowledge", Strateg. Manag. J., vol. 34, n. ${ }^{\circ}$ 2, pp. 1513-1530, 2013. [Online]. doi: https://doi.org/10.1002/smj.2080

[50] P. Puranam, O. Alexy y M. Reitzig, "What's 'New' About New Forms of Organizing?", Acad. Manag. Rev., vol. 39, n. ${ }^{\circ}$, pp. 162-180, 2014. [Online]. doi: https://doi.org/10.5465/amr.2011.0436

[51] S. Furnari, "Interstitial Spaces: Microinteraction Settings and the Genesis of New Practices between Institutional Fields", Acad. Manag. Rev., vol. 39, n. ${ }^{\circ} 4$, pp. 439-462, 2014. [Online]. doi: https://doi. org/10.5465/amr.2012.0045

[52] B. G. King, E. S. Clemens y M. Fry, "Identity Realization and Organizational Forms: Differentiation and Consolidation of Identities among Arizona's Charter Schools", Organ. Sci., vol. 22, n. ${ }^{\circ}$ 3, pp. 554-572, 2011. [Online]. doi: https://doi.org/10.1287/orsc. 1100.0548

[53] S. E. Seibert, L. D. Sargent, M. L. Kraimer y K. Kiazad, "Linking Developmental Experiences to Leader Effectiveness and Promotability: the Mediating Role of Leadership SelfEfficacy and Mentor Network", Pers. Psychol., 2015. [Online]. doi: https://doi.org/10.1111/ peps. 12145

[54] D. E. Bailey y S. R. Barley, "TeachingLearning Ecologies: Mapping the Environment to Structure through Action", Organ. Sci., vol. 22, n. ${ }^{\circ}$ 1, pp. 262-285, 2011. [Online]. doi: https://doi.org/10.1287/orsc.1090.0511

[55] X. Song, W. Shi, Y. Ma y C. Yang, "Impact of informal networks on opinion dynamics in hierarchically formal organization", Phys. A Stat. Mech. its Appl., vol. 436, pp. 916-924, 2015. [Online]. doi: https:// doi.org/10.1016/j.physa.2015.05.051

[56] J. J. Reuer, T. W. Tong, B. B. Tyler y A. Ariño, "Executive preferences for governance modes and exchange partners: an Information economics perspective", Strateg. Manag. J., vol. 34, n. ${ }^{\circ}$ 2, pp. 1104-1122, 2013. [Online]. doi: https://doi.org/10.1002/smj.2064

[57] T. D. Allen, R. C. Johnson, K. M. Kiburz y K. M. Shockley, "WorkFamily Conflict and Flexible Work
Arrangements: Deconstructing Flexibility", Pers. Psychol., vol. 66, n. ${ }^{\circ}$ 2, pp. 345-376, 2013. [Online]. doi: https://doi.org/10.1111/peps.12012

[58] F. A. Csaszar, "Organizational structure as a determinant of performance: evidence from mutual funds", Strateg. Manag. J., vol. 33, n. ${ }^{\circ}$ 2, pp. 611-632, 2012. [Online]. Disponible en https://papers.ssrn. com/sol3/papers.cfm?abstract_id=1281559

[59] V. Salas Fumás, C. Sáenz Royo y Á. Lozano Rojo, "Organisational structure and performance of consensus decisions through mutual influences: A computer simulation approach", Decis. Support Syst., vol. 86, pp. 61-72, 2015. [Online]. doi: https://doi. org/10.1016/j.dss.2016.03.008

[60] C. Fang and M. A. Schilling, "When hubs forget, lie, and play favorites: interpersonal network structure, information distortion, and organizational learning", Strateg. Manag. J., vol. 35, n. ${ }^{\circ}$ 2, pp. 974-994, 2014. [Online]. doi: https://doi.org/10.1002/smj.2142

[61] J. Qiu y Z. Lin, "A framework for exploring organizational structure in dynamic social networks", Decis. Support Syst., vol. 51, n. ${ }^{\circ} 4$, pp. 760-771, 2011. [Online]. doi: https://doi.org/10.1016/j.dss.2011.01.011

[62] S. Leroy, A. J. Shipp, S. Blount y J. G. Lic [Online], "Synchrony Preference: Why Some People Go With the Flow and Some Don't", Pers. Psychol., vol. 68, n. ${ }^{\circ} 4$, pp. 759-809, 2015. [Online]. doi: https://doi. org/10.1111/peps.12093

[63] W. F. Boh y S. S. Wong, "Managers versus coworkers as referents: Comparing social influence effects on within and outside subsidiary knowledge sharing", Organ. Behav. Hum. Decis. Process, vol. 126, pp. 1-17, 2015. [Online]. doi: https://doi.org/10.1016/j. obhdp.2014.09.008

[64] J. R. Methot, J. A. Lepine, N. P. Podsakoff, y J. S. Christian, "Are Workplace Friendships a Mixed Blessing? Exploring Tradeoffs of Multiplex Relationships and their Associations with Job Performance", Pers. Psychol., vol. 69, n. ${ }^{\circ}$ 2, pp. 311-355, 2016. [Online]. doi: http://dx.doi.org/10.1111/peps.12109

[65] J. R. Hollenbeck, A. P. J. Ellis, S. E. Humphrey, A. S. Garza y D. R. Ilgen, "Asymmetry in structural adaptation: The differential impact of centralizing versus decentralizing team decision making structures", Organ. Behav. Hum. Decis. Process. vol. 114, n. ${ }^{\circ} 1$, pp. 64-74, 2011. [Online]. doi: https://doi.org/10.1016/j. obhdp.2010.08.003

[66] G. Soda y A. Zaheer, "A network perspective on organizational architecture: performance effects of the interplay of formal and informal organization", Strateg. Manag. J., vol. 33, n. ${ }^{\circ}$ 2, pp. 751-771, 2012. [Online]. doi: https://doi.org/10.1002/smj.1966 